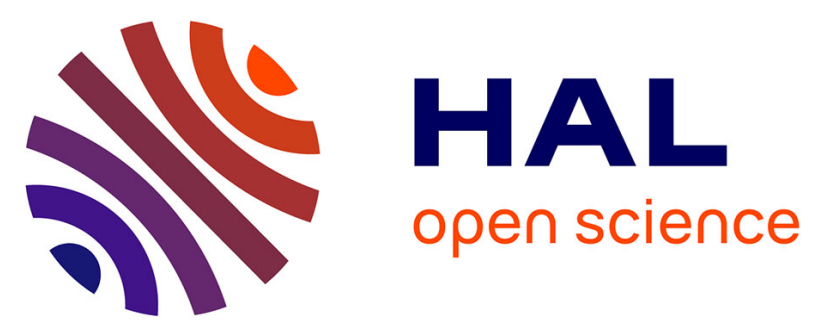

\title{
Completing an experimental nondepolarizing Mueller matrix whose column or row is missing Completing an experimental nondepolarizing Mueller matrix whose column or row is missing
}

Razvigor Ossikovski, Oriol Arteaga

\section{To cite this version:}

Razvigor Ossikovski, Oriol Arteaga. Completing an experimental nondepolarizing Mueller matrix whose column or row is missing Completing an experimental nondepolarizing Mueller matrix whose column or row is missing. Journal of Vacuum Science \& Technology B Microelectronics and Nanometer Structures, 2019, 37, 10.1116/1.5120342 . hal-02436934

HAL Id: hal-02436934

https://hal-polytechnique.archives-ouvertes.fr/hal-02436934

Submitted on 13 Jan 2020

HAL is a multi-disciplinary open access archive for the deposit and dissemination of scientific research documents, whether they are published or not. The documents may come from teaching and research institutions in France or abroad, or from public or private research centers.
L'archive ouverte pluridisciplinaire HAL, est destinée au dépôt et à la diffusion de documents scientifiques de niveau recherche, publiés ou non, émanant des établissements d'enseignement et de recherche français ou étrangers, des laboratoires publics ou privés. 
Completing an experimental nondepolarizing Mueller matrix whose column or row is missing

Razvigor Ossikovski, and Oriol Arteaga

Citation: Journal of Vacuum Science \& Technology B 37, 052905 (2019); doi: 10.1116/1.5120342

View online: https://doi.org/10.1116/1.5120342

View Table of Contents: https://avs.scitation.org/toc/jvb/37/5

Published by the American Vacuum Society

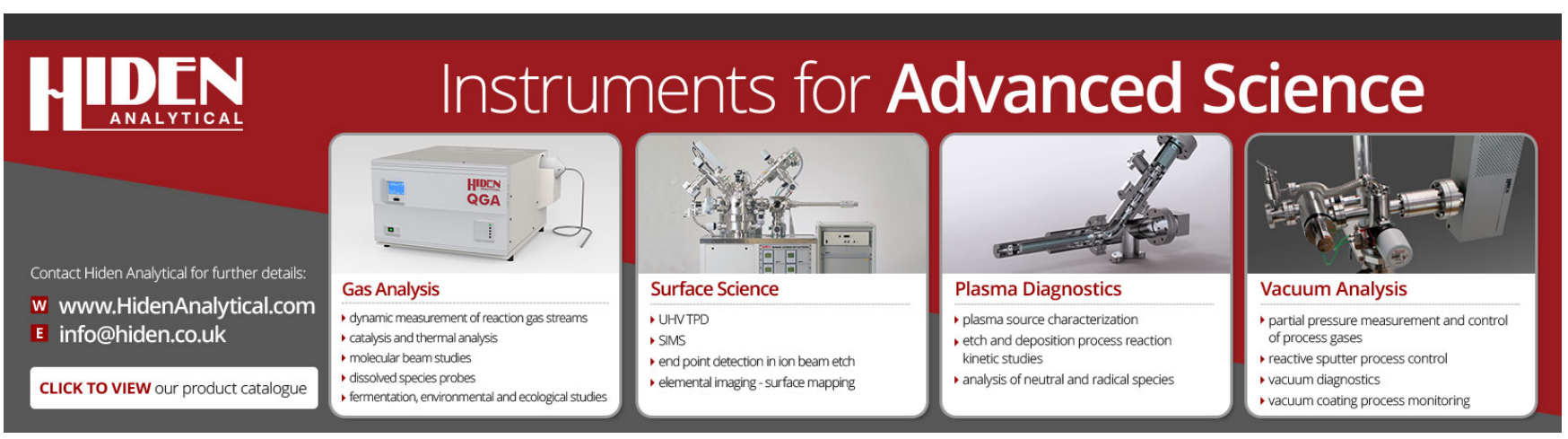




\title{
Completing an experimental nondepolarizing Mueller matrix whose column or row is missing
}

\author{
Razvigor Ossikovski ${ }^{1, a)}$ and Oriol Arteaga ${ }^{1,2}$ \\ ${ }^{1}$ LPICM, CNRS, Ecole Polytechnique, Institut Polytechnique de Paris, route de Saclay, \\ 91128 Palaiseau, France \\ ${ }^{2}$ Dep. de Física Aplicada, IN2UB, Feman Group, Universitat de Barcelona, C/Martí i Franquès 1, \\ Barcelona 08030, Spain
}

(Received 17 July 2019; accepted 4 September 2019; published 26 September 2019)

\begin{abstract}
The authors report on an algebraic procedure, allowing for the completion of an experimental nondepolarizing Mueller matrix with a column or a row missing to a full 16-element one. The method provides a closed-form solution for the missing column or row and is illustrated on single-wavelength, spectroscopic, and imaging experimental examples. Published by the AVS.

https://doi.org/10.1116/1.5120342
\end{abstract}

\section{INTRODUCTION}

The real $4 \times 4$ Mueller matrix $\mathbf{M}$ is the most general phenomenological descriptor of the linear interaction of a medium or an optical arrangement with polarized light, represented in the form of the incident and emerging Stokes vectors. ${ }^{1}$ If no loss of spatial or temporal coherence takes place during the measurement, the resulting experimental Mueller matrix $\mathbf{M}_{\mathrm{nd}}$ is termed nondepolarizing since it transforms any totally polarized incident Stokes vector into a totally polarized emerging one. ${ }^{1,2}$ The matrix $\mathbf{M}_{\mathrm{nd}}$ is then equivalent to a complex $2 \times 2$ Jones matrix $\mathbf{J}$ (wherefrom its alternative name of the Mueller-Jones matrix), which means that its 16 real elements are not independent but are rather functions of $2 \times 2 \times 2-1=7$ parameters. (Note that $\mathbf{J}$ and $\mathbf{J} e^{i \varphi}$, where $\varphi$ is a real "absolute" phase, result in one and the same $\mathbf{M}_{\mathrm{nd}}$, wherefrom the subtraction of the unit.) In other words, there exist $16-7=9$ relationships ${ }^{3}$ between the elements of $\mathbf{M}_{\mathrm{nd}}$. Conversely, in the presence of depolarization, ${ }^{4}$ i.e., if spatial or spectral coherence is either partially or totally lost during the experiment, there is no one-to-one correspondence between $\mathbf{M}$ and $\mathbf{J}$ ( $\mathbf{J}$ can be then defined only in a statistical sense $\mathrm{e}^{4}$ ) so that all 16 elements of $\mathbf{M}$ are independent.

The fact that the nondepolarizing Mueller matrix $\mathbf{M}_{n d}$ depends on only 7 rather than on a total of 16 parameters suggests that, in principle, one should be able to complete it to its full form even if one has measured it only partially. Indeed, conventional partial Mueller polarimeter designs provide experimentally either 9 (when both a column and a row are missing) or 12 (when a column or a row is missing) elements of M. ${ }^{5,6}$ In the first case, it has been shown that the completion problem for a partial nondepolarizing Mueller matrix $\mathbf{M}_{\mathrm{nd}}$ features exactly two distinct solutions, ${ }^{7}$ whereas in the second one, the solution is unique. ${ }^{8}$ In both cases, the completion of the partial 9- or 12-element $\mathbf{M}_{\mathrm{nd}}$ to a full 16-element one is based on the fact that its associated

Note: This paper is part of the Conference Collection: 8th International Conference on Spectroscopic Ellipsometry 2019, ICSE.

${ }^{\text {a)} E l e c t r o n i c ~ m a i l: ~ r a z v i g o r . o s s i k o v s k i @ p o l y t e c h n i q u e . e d u ~}$ covariance matrix ${ }^{2} \mathbf{H}_{\mathrm{nd}}$ must be of unit rank. This involves the conversion of the elements of $\mathbf{M}_{\mathrm{nd}}$ into those of $\mathbf{H}_{\mathrm{nd}}$ and back, and furthermore requires making a judicious choice of equations from an overdetermined set, thus making only partial use of the information contained in $\mathbf{H}_{\mathrm{nd}}$.

An alternative approach to the recovery of the full $\mathbf{M}_{\mathrm{nd}}$ from its partial experimental counterpart would consist in exploiting the nine relationships existing between its elements. Since the uniqueness of the solution is ensured only in the 12-element partial nondepolarizing Mueller matrix case, the alternative approach will be applied to it. Furthermore, this case is of significant experimental importance since 12 -element partial Mueller polarimeters ${ }^{9}$ are commonly used to characterize anisotropic nondepolarizing samples by performing so-called "generalized ellipsometry."10 Although measurements using complete Mueller matrix polarimeters are relatively widespread, 12-element partial systems still retain strong scientific and commercial interest essentially because of their simpler optical layout usually offering a wider spectral range, increased sensitivity, and better adaptability to imaging operation. Therefore, the development of a computationally efficient closed-form algebraic procedure for the recovery of the full $\mathbf{M}_{\text {nd }}$ from its 12-element experimental counterpart having a column or a row missing, without resorting to the evaluation of its associated covariance matrix $\mathbf{H}_{\mathrm{nd}}$ or of its equivalent Jones matrix $\mathbf{J}$, is of definite practical interest.

\section{ALGEBRAIC PROCEDURE}

It has been established independently by several authors $^{11-14}$ that the set of relationships ${ }^{15,16}$ relating the elements of a nondepolarizing Mueller matrix can be cast into the following matrix form:

$$
\mathbf{M}^{T} \mathbf{G M}=\sqrt{|\mathbf{M}|} \mathbf{G},
$$

in which $\mathbf{G}=\operatorname{diag}\left(\begin{array}{llll}1 & -1 & -1 & -1\end{array}\right)$ is the Minkowski metric (the notation $|\cdots|$ stands for determinant and the superscript " $T$ " denotes transposition). (For simplicity, from now on, we shall write simply $\mathbf{M}$ instead of $\mathbf{M}_{\text {nd }}$ for a nondepolarizing Mueller matrix.) 
In what follows, we shall assume that the 12-element partial nondepolarizing Mueller matrix $\mathbf{M}$ has its last (i.e., fourth) column missing (i.e., undetermined experimentally). If instead the second or the third column of $\mathbf{M}$ is missing, then it can be permuted with the fourth one; the recovery procedure is applied and the final result is permuted back. (Notice that the first column or row of $\mathbf{M}$ is always measured by partial polarimeters.) Alternatively, if a row instead of a column is missing, then one can apply the procedure to the transpose $\mathbf{M}^{T}$ of $\mathbf{M}$ and retranspose the final result. Under the above assumption, the 12-element partial $\mathbf{M}$ can be partitioned columnwise,

$$
\mathbf{M}=\left[\begin{array}{llll}
\mathbf{m}_{1} & \mathbf{m}_{2} & \mathbf{m}_{3} & \mathbf{v}
\end{array}\right],
$$

where $\mathbf{m}_{k}=\left[\begin{array}{llll}M_{1 k} & M_{2 k} & M_{3 k} & M_{4 k}\end{array}\right]^{T}, k=1,2,3$, is its $k$ th column and $\mathbf{v}=\left[\begin{array}{llll}v_{1} & v_{2} & v_{3} & v_{4}\end{array}\right]^{T}$ is its unknown last column $\left(M_{i k}, i=1,2,3,4, k=1,2,3\right.$ are the 12 known elements of M). Substituting Eq. (2) into Eq. (1) results in

$$
\begin{gathered}
{\left[\begin{array}{cccc}
\mathbf{m}_{1}^{T} \mathbf{G} \mathbf{m}_{1} & \mathbf{m}_{1}^{T} \mathbf{G} \mathbf{m}_{2} & \mathbf{m}_{1}^{T} \mathbf{G} \mathbf{m}_{3} & \mathbf{m}_{1}^{T} \mathbf{G} \mathbf{v} \\
\mathbf{m}_{2}^{T} \mathbf{G} \mathbf{m}_{1} & \mathbf{m}_{2}^{T} \mathbf{G} \mathbf{m}_{2} & \mathbf{m}_{2}^{T} \mathbf{G} \mathbf{m}_{3} & \mathbf{m}_{2}^{T} \mathbf{G} \mathbf{v} \\
\mathbf{m}_{3}^{T} \mathbf{G} \mathbf{m}_{1} & \mathbf{m}_{3}^{T} \mathbf{G} \mathbf{m}_{2} & \mathbf{m}_{3}^{T} \mathbf{G} \mathbf{m}_{3} & \mathbf{m}_{3}^{T} \mathbf{G} \mathbf{v} \\
\mathbf{v}^{T} \mathbf{G} \mathbf{m}_{1} & \mathbf{v}^{T} \mathbf{G} \mathbf{m}_{2} & \mathbf{v}^{T} \mathbf{G} \mathbf{m}_{3} & \mathbf{v}^{T} \mathbf{G} \mathbf{v}
\end{array}\right]} \\
=\sqrt{|\mathbf{M}|}\left[\begin{array}{cccc}
1 & 0 & 0 & 0 \\
0 & -1 & 0 & 0 \\
0 & 0 & -1 & 0 \\
0 & 0 & 0 & -1
\end{array}\right] .
\end{gathered}
$$

By equating the last columns of both sides of Eq. (3) one readily obtains the following set of three equations for the unknown column $\mathbf{v}$ :

$$
\mathbf{m}_{1}^{T} \mathbf{G} \mathbf{v}=\mathbf{m}_{2}^{T} \mathbf{G} \mathbf{v}=\mathbf{m}_{3}^{T} \mathbf{G} \mathbf{v}=0 .
$$

These equations can be grouped into the single matrix equation

$$
\mathbf{A} \mathbf{v}=\left[\begin{array}{l}
\mathbf{m}_{1}^{T} \mathbf{G} \\
\mathbf{m}_{2}^{T} \mathbf{G} \\
\mathbf{m}_{3}^{T} \mathbf{G}
\end{array}\right] \mathbf{v}=\left[\begin{array}{llll}
M_{11} & -M_{21} & -M_{31} & -M_{41} \\
M_{12} & -M_{22} & -M_{32} & -M_{42} \\
M_{13} & -M_{23} & -M_{33} & -M_{43}
\end{array}\right]\left[\begin{array}{c}
v_{1} \\
v_{2} \\
v_{3} \\
v_{4}
\end{array}\right]=\mathbf{0}
$$

where $\mathbf{A}$ is an auxiliary $3 \times 4$ matrix. It is well known from linear algebra that the solution of Eq. (5) is given by

$$
\mathbf{v}=v_{0}\left[\begin{array}{llll}
A_{1} & A_{2} & A_{3} & A_{4}
\end{array}\right]^{T},
$$

where $v_{0}$ is a (real) proportionality factor and $A_{m}, m=1,2$,
3, 4, are the adjuncts (i.e., the signed minors) obtained by striking out the $m$ th column of $\mathbf{A}$,

$$
\begin{aligned}
& A_{1}=(-1)^{1}\left|\begin{array}{lll}
-M_{21} & -M_{31} & -M_{41} \\
-M_{22} & -M_{32} & -M_{42} \\
-M_{23} & -M_{33} & -M_{43}
\end{array}\right|=\left|\begin{array}{lll}
M_{21} & M_{22} & M_{23} \\
M_{31} & M_{32} & M_{33} \\
M_{41} & M_{42} & M_{43}
\end{array}\right|, \\
& A_{2}=(-1)^{2}\left|\begin{array}{lll}
M_{11} & -M_{31} & -M_{41} \\
M_{12} & -M_{32} & -M_{42} \\
M_{13} & -M_{33} & -M_{43}
\end{array}\right|=\left|\begin{array}{lll}
M_{11} & M_{12} & M_{13} \\
M_{31} & M_{32} & M_{33} \\
M_{41} & M_{42} & M_{43}
\end{array}\right|,
\end{aligned}
$$

$$
\begin{aligned}
& A_{3}=(-1)^{3}\left|\begin{array}{lll}
M_{11} & -M_{21} & -M_{41} \\
M_{12} & -M_{22} & -M_{42} \\
M_{13} & -M_{23} & -M_{43}
\end{array}\right|=-\left|\begin{array}{lll}
M_{11} & M_{12} & M_{13} \\
M_{21} & M_{22} & M_{23} \\
M_{41} & M_{42} & M_{43}
\end{array}\right|, \\
& A_{4}=(-1)^{4}\left|\begin{array}{lll}
M_{11} & -M_{21} & -M_{31} \\
M_{12} & -M_{22} & -M_{32} \\
M_{13} & -M_{23} & -M_{33}
\end{array}\right|=\left|\begin{array}{lll}
M_{11} & M_{12} & M_{13} \\
M_{21} & M_{22} & M_{23} \\
M_{31} & M_{32} & M_{33}
\end{array}\right| .
\end{aligned}
$$

Notice that the minors appearing in the right-hand sides of Eqs. (7a)-(7d) can be obtained simply by striking out the $m$ th row and the fourth column of $\mathbf{M}$, i.e., without the need for constructing the auxiliary matrix $\mathbf{A}$.

The absolute value $\left|v_{0}\right|$ of the proportionality factor $v_{0}$ entering Eq. (6) can be determined from the equalities

$$
\mathbf{v}^{T} \mathbf{G} \mathbf{v}=-\mathbf{m}_{1}^{T} \mathbf{G} \mathbf{m}_{1}=\mathbf{m}_{2}^{T} \mathbf{G} \mathbf{m}_{2}=\mathbf{m}_{3}^{T} \mathbf{G} \mathbf{m}_{3}
$$

obtained by equating the diagonal elements of both sides of Eq. (3). After substitution of Eq. (6) into Eq. (8), one gets three equivalent expressions for $\left|v_{0}\right|$,

$$
\begin{aligned}
\left|v_{0}\right| & =\sqrt{\frac{\mathbf{m}_{1}^{T} \mathbf{G} \mathbf{m}_{1}}{A_{4}^{2}+A_{3}^{2}+A_{2}^{2}-A_{1}^{2}}}=\sqrt{\frac{-\mathbf{m}_{2}^{T} \mathbf{G} \mathbf{m}_{2}}{A_{4}^{2}+A_{3}^{2}+A_{2}^{2}-A_{1}^{2}}} \\
& =\sqrt{\frac{-\mathbf{m}_{3}^{T} \mathbf{G} \mathbf{m}_{3}}{A_{4}^{2}+A_{3}^{2}+A_{2}^{2}-A_{1}^{2}}},
\end{aligned}
$$

where $\mathbf{m}_{k}^{T} \mathbf{G} \mathbf{m}_{k}=M_{1 k}^{2}-M_{2 k}^{2}-M_{3 k}^{2}-M_{4 k}^{2}, \quad k=1, \quad 2, \quad 3$. On experimental data, one can average advantageously the three theoretically equal products $\mathbf{m}_{1}^{T} \mathbf{G} \mathbf{m}_{1},-\mathbf{m}_{2}^{T} \mathbf{G} \mathbf{m}_{2}$, and $-\mathbf{m}_{3}^{T} \mathbf{G} \mathbf{m}_{3}$ for better noise resilience, i.e., rather use the following expression:

$$
\left|v_{0}\right|=\sqrt{\frac{\mathbf{m}_{1}^{T} \mathbf{G} \mathbf{m}_{1}-\mathbf{m}_{2}^{T} \mathbf{G} \mathbf{m}_{2}-\mathbf{m}_{3}^{T} \mathbf{G} \mathbf{m}_{3}}{3\left(A_{4}^{2}+A_{3}^{2}+A_{2}^{2}-A_{1}^{2}\right)}}=\sqrt{\frac{M_{11}^{2}-M_{21}^{2}-M_{31}^{2}-M_{41}^{2}-M_{12}^{2}+M_{22}^{2}+M_{32}^{2}+M_{42}^{2}-M_{13}^{2}+M_{23}^{2}+M_{33}^{2}+M_{43}^{2}}{3\left(A_{4}^{2}+A_{3}^{2}+A_{2}^{2}-A_{1}^{2}\right)},}
$$


instead of Eq. (9a).

Clearly, Eq. (6) yields two solutions for the unknown last column of $\mathbf{M}, \mathbf{v}_{+}$, and $\mathbf{v}_{-}=-\mathbf{v}_{+}$,

$$
\mathbf{v}_{ \pm}= \pm\left|v_{0}\right|\left[\begin{array}{llll}
A_{1} & A_{2} & A_{3} & A_{4}
\end{array}\right]^{T}
$$

where $\left|v_{0}\right|$ is given by Eqs. (9a) and (9b). To pick up the correct solution use should be made of the fact that the determinant $|\mathbf{M}|$ of any nondepolarizing Mueller matrix $\mathbf{M}$ is nonnegative, ${ }^{13}$

$$
\begin{aligned}
|\mathbf{M}| & =\left|\begin{array}{llll}
M_{11} & M_{12} & M_{13} & v_{1} \\
M_{21} & M_{22} & M_{23} & v_{2} \\
M_{31} & M_{32} & M_{33} & v_{3} \\
M_{41} & M_{42} & M_{43} & v_{4}
\end{array}\right| \\
& =-A_{1} v_{1}+A_{2} v_{2}+A_{3} v_{3}+A_{4} v_{4} \geq 0 .
\end{aligned}
$$

The last expression in Eq. (11) is the expansion of the determinant on the elements of the last column of $\mathbf{M}$, involving the adjuncts from Eqs. (7a)-(7d). Substitution of the first of Eq. (9a) into Eq. (10) followed by that of Eq. (10) into Eq. (11) yields

$$
|\mathbf{M}|= \pm \sqrt{\frac{\mathbf{m}_{1}^{T} \mathbf{G} \mathbf{m}_{1}}{A_{4}^{2}+A_{3}^{2}+A_{2}^{2}-A_{1}^{2}}}\left(-A_{1}^{2}+A_{2}^{2}+A_{3}^{2}+A_{4}^{2}\right) \geq 0 .
$$

Since $\mathbf{m}_{1}^{T} \mathbf{G} \mathbf{m}_{1}=M_{11}^{2}-M_{21}^{2}-M_{31}^{2}-M_{41}^{2} \geq 0$ (because the first column of any Mueller matrix is necessarily a Stokes vector), then $A_{4}^{2}+A_{3}^{2}+A_{2}^{2}-A_{1}^{2}=-A_{1}^{2}+A_{4}^{2}+A_{3}^{2}+A_{2}^{2} \geq 0$ (because the fraction under the square root must be nonnegative). Therefore, the correct solution is $\mathbf{v}=\mathbf{v}_{+}$since the factor $-A_{1}^{2}+A_{4}^{2}+A_{3}^{2}+A_{2}^{2}$ multiplying the square root in Eq. (12) is non-negative. Consequently, the missing last column $\mathbf{v}$ of $\mathbf{M}$ is determined uniquely. This completes the procedure.

\section{EXPERIMENTAL VALIDATION}

The following experimental Mueller matrix

$$
\mathbf{M}_{\exp }=\left[\begin{array}{cccc}
1.000 & -0.440 & 0.421 & 0.117 \\
-0.434 & 0.767 & -0.113 & -0.449 \\
-0.423 & 0.113 & -0.882 & 0.013 \\
0.119 & -0.448 & -0.007 & -0.657
\end{array}\right]
$$

is that of a diffraction grating measured at the wavelength of $400 \mathrm{~nm}$ in reflection configuration at an incidence angle of $75^{\circ}$ by using a UV-visible spectroscopic complete Mueller polarimeter. ${ }^{17}$ The grating whose structure is described in detail elsewhere ${ }^{18}$ was rotated at $45^{\circ}$ with respect to the plane of incidence so that all Mueller matrix elements feature nonzero values. Assuming that only the first three columns of $\mathbf{M}_{\exp }$ were measured (on a partial
Mueller polarimeter) and applying Eqs. (7), (9b), and (10) yields the following recovered complete Mueller matrix $\mathbf{M}_{\mathrm{rec}}$ :

$$
\mathbf{M}_{\mathrm{rec}}=\left[\begin{array}{cccc}
1.000 & -0.440 & 0.421 & 0.113 \\
-0.434 & 0.767 & -0.113 & -0.448 \\
-0.423 & 0.113 & -0.882 & 0.009 \\
0.119 & -0.448 & -0.007 & -0.653
\end{array}\right] .
$$

Comparison of $\mathbf{M}_{\exp }$ from Eq. (13) and $\mathbf{M}_{\mathrm{rec}}$ from Eq. (14) indicates successful recovery of the last column, the largest absolute error being 0.004 , to be compared to the typical $0.5 \%$ experimental accuracy of the Mueller polarimeter used. ${ }^{17}$ This example demonstrates the applicability of the procedure to experimental Mueller matrices with nonzero elements in their two $2 \times 2$ off-diagonal blocks, typical of patterned anisotropic samples characterized in the conical diffraction mode.

To study the potential spectral dependence of the quality of recovery, the procedure was applied over the entire experimentally available spectral region ranging from 240 to $650 \mathrm{~nm}$. The result is shown in Fig. 1 .

The calculated values of the elements of the last column of the Mueller matrix virtually overlapped their experimental counterparts so that the latter are not shown in the figure. The recovered elements obey the symmetry relations $M_{14}=M_{41}$, $M_{24}=M_{42}$, and $M_{34}=-M_{43}$ expected for a sample whose polarimetric response is rotationally invariant with respect to a $180^{\circ}$-rotation about its normal. ${ }^{3,8}$ It should be emphasized that the recovery procedure does not assume the validity of any symmetry relations; indeed, the latter are violated by the presence of slight asymmetries in the pattern profiles, as has been shown both theoretically ${ }^{19}$ and experimentally. ${ }^{20-23}$ The quality of recovery of the last column of the Mueller matrix is spectrally uniform. The average error (rms value) per element is 0.0027 and is thus lower than the experimental accuracy. This value is comparable to that obtained by applying either of the covariance-matrix-based methods (analytical or numerical) from Ref. 8. However, the uniformity of the present procedure is better: it features a maximum error of 0.017 over the entire spectrum as evidenced by Fig. 1, whereas that of the covariance-matrix-based methods attains 0.068 .

The closed-form expressions of the recovery procedure turn out particularly useful in imaging applications, where high-resolution detector arrays generate a large number of experimental data points in the form of (complete or partial) Mueller matrices, and consequently, the image processing time is an essential parameter. As an example, we applied the procedure on the Mueller matrix image of a thin polycrystalline sample of banded mannitol spherulites dyed with Chicago sky blue 6B tetrasodium salt that crystallize in two distinct polymorphs (more details can be found in Ref. 24). The measurement was performed on an imaging Mueller matrix microscope $\mathrm{e}^{25}$ at a wavelength of $630 \mathrm{~nm}$. Figure 2 shows both the complete experimental Mueller matrix as well as the last column recovered by using the information from the 12 elements of the first three columns. The 

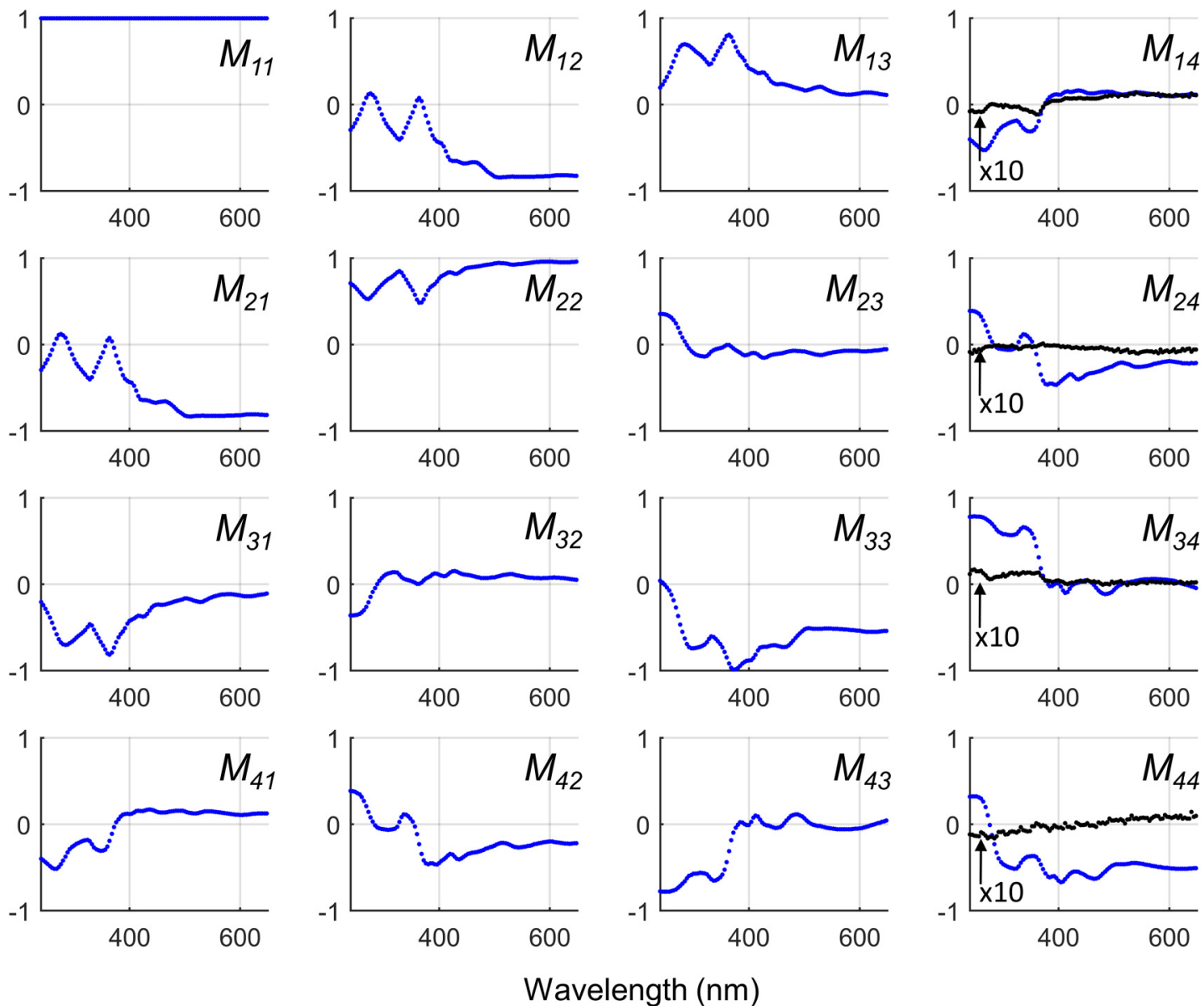

Wavelength $(\mathrm{nm})$

FIG. 1. Recovered complete spectroscopic Mueller matrix of the diffraction grating from the 12-element partial one with the last column assumed missing. The difference (multiplied by ten for better visibility) between measured and recovered last column elements is likewise shown.

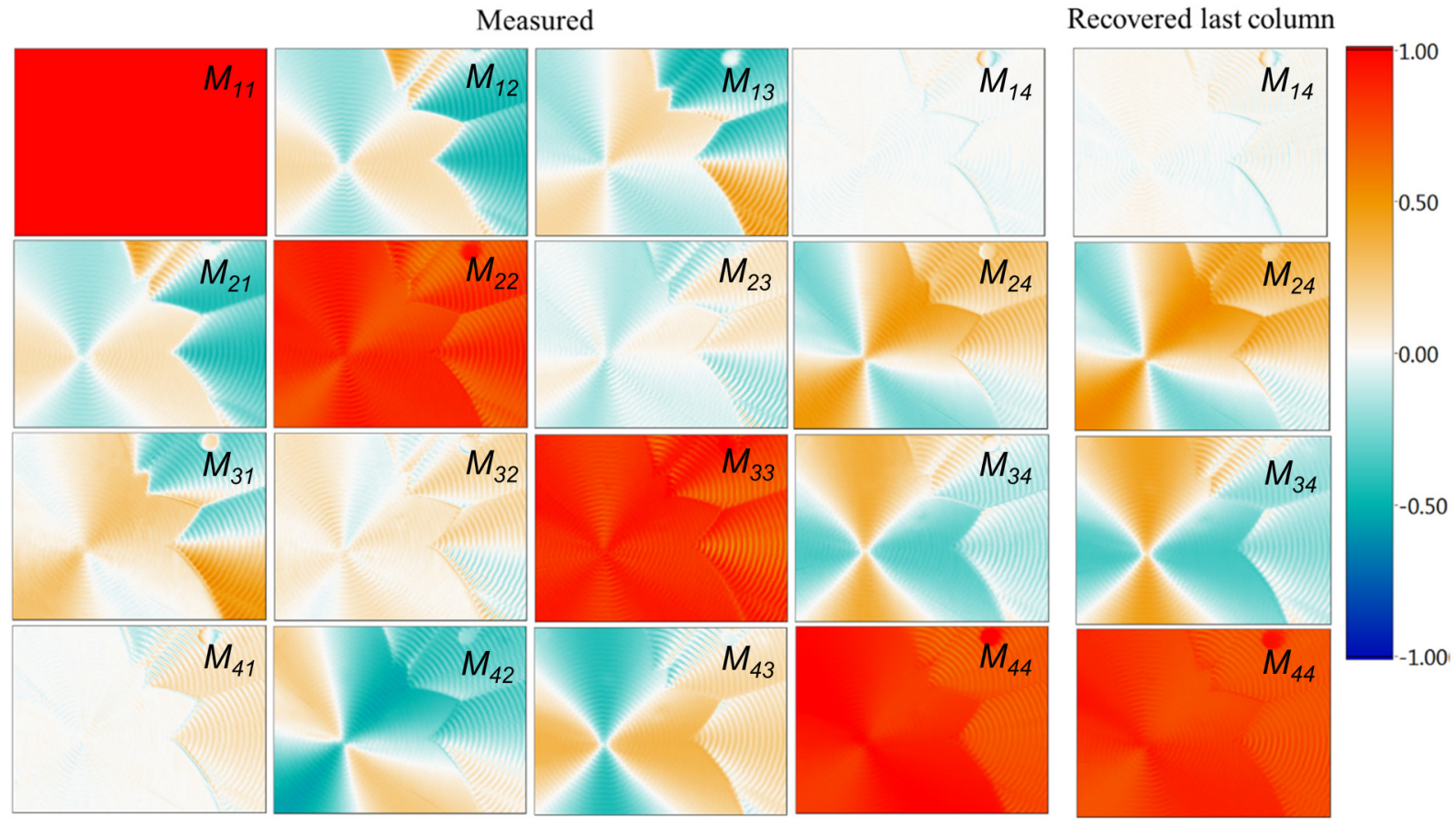

Fig. 2. Experimental Mueller matrix image of a thin polycrystalline sample of dyed banded mannitol spherulites and its recovered last column from its three-column, 12-element partial counterpart. 
measured and recovered last columns compare very favorably; in particular, the quality of recovery is spatially uniform and reproduces successfully all features originally resolved by the microscope.

These experimental results qualify the algebraic procedure for practical use.

Before concluding, it should be emphasized that recovery is feasible only if the experimental Mueller matrix $\mathbf{M}$ is nondepolarizing. If no preliminary information on $\mathbf{M}$ is available, one can still apply the procedure and evaluate $a$ posteriori the eigenvalues of the covariance matrix ${ }^{2} \mathbf{H}$ associated with the recovered complete $\mathbf{M}$. If the second largest eigenvalue of $\mathbf{H}$ is lower than the experimental accuracy, then $\mathbf{H}$ is of unit rank and the procedure is applicable. ${ }^{7}$ If this is not the case, one should resort to more complex covariance-matrix-based procedures. ${ }^{8}$ Alternatively, one may evaluate the depolarization index (or the degree of polarimetric purity $)^{4}$ of the recovered $\mathbf{M}$; a value equal to the unit within the experimental error is a warrant of successful recovery.

\section{SUMMARY AND CONCLUSIONS}

An algebraic procedure providing closed-form expressions for the elements of a missing column or row of a partially measured 12-element nondepolarizing experimental Mueller matrix has been described. When applied on experimental data, the procedure results in generally lower absolute errors compared to those of previously developed recovery methods. The procedure is believed to be of use to experimentalists willing to recover the complete nondepolarizing Mueller matrix from a partial polarimetry experiment.

\section{ACKNOWLEDGMENTS}

This work was partially funded by the European Commission (Polarsense, No. MSCA-IF-2017-793774).
The authors are grateful to their colleague E. Garcia-Caurel for having provided them with the diffraction grating sample.

${ }^{1}$ R. M. A. Azzam and N. M. Bashara, Ellipsometry and Polarized Light (North Holland, Amsterdam, 1987).

${ }^{2}$ J. J. Gil and R. Ossikovski, Polarized Light and the Mueller Matrix Approach (CRC, Boca Raton, 2016).

${ }^{3}$ H. C. van de Hulst, Light Scattering by Small Particles (Dover, New York, 1981).

${ }^{4}$ C. Brosseau, Fundamentals of Polarized Light. A Statistical Optics Approach (Wiley, New York, 1998).

${ }^{5}$ P. S. Hauge, Surf. Sci. 96, 108 (1980).

${ }^{6} \mathrm{R}$. A. Chipman, in Handbook of Optics II, edited by M. Bass (McGraw Hill, New York, 1995), pp. 22.1-22.38.

${ }^{7}$ R. Ossikovski and O. Arteaga, J. Opt. Soc. Am. A 36, 403 (2019).

${ }^{8}$ O. Arteaga and R. Ossikovski, J. Opt. Soc. Am. A 36, 416 (2019).

${ }^{9}$ G. E. Jellison, Jr. and F. A. Modine, Appl. Opt. 36, 8190 (1997).

${ }^{10}$ R. M. A. Azzam and N. M. Bashara, J. Opt. Soc. Am. A 64, 128 (1974).

${ }^{11}$ R. Barakat, Opt. Commun. 38, 159 (1981).

${ }^{12}$ R. Simon, Opt. Commun. 42, 293 (1982).

${ }^{13}$ Z.-F. Xing, J. Mod. Opt. 39, 461 (1992).

${ }^{14}$ J. W. Hovenier, Appl. Opt. 33, 8318 (1994).

${ }^{15}$ K. D. Abhyankar and A. L. Fymat, J. Math. Phys. 10, 1935 (1969).

${ }^{16}$ E. S. Fry and G. W. Kattawar, Appl. Opt. 20, 2811 (1981).

${ }^{17}$ O. Arteaga, J. Freudenthal, B. Wang, and B. Kahr, Appl. Opt. 51, 6805 (2012).

${ }^{18}$ M. Foldyna, E. Garcia-Caurel, R. Ossikovski, A. De Martino, and J. J. Gil, Opt. Express 17, 19 (2009).

${ }^{19}$ T. Novikova, R. Ossikovski, A. De Martino, and B. Drévillon, Eur. J. Phys. Appl. Phys. 31, 63 (2005).

${ }^{20}$ T. Novikova, P. Bulkin, V. Popov, B. Haj Ibrahim, and A. De Martino, J. Vac. Sci. Technol. B 29, 051804 (2011).

${ }^{21}$ C. Fallet, T. Novikova, M. Foldyna, S. Manhas, B. Haj Ibrahim, A. De Martino, C. Vannuffel, and C. Constancias, J. Micro/Nanolith. MEMS MOEMS 10, 033017 (2011).

${ }^{22}$ D. Dixit et al., J. Micro/Nanolith. MEMS MOEMS 15, 014004 (2016).

${ }^{23}$ D. Dixit, N. Keller, Y. Lifshitz, T. Kagalwala, A. Elia, V. Todi, J. Fronheiser, and A. Vaid, J. Micro/Nanolith. MEMS MOEMS 17, 034001 (2018).

${ }^{24}$ X. Cui, S. M. Nichols, O. Arteaga, J. Freudenthal, F. Paula, A. G. Shtukenberg, and B. Kahr, J. Am. Chem. Soc. 138, 12211 (2016).

${ }^{25}$ O. Arteaga, M. Baldrís, J. Antó, A. Canillas, E. Pascual, and E. Bertran, Appl. Opt. 53, 2236 (2014). 